\title{
Problem of Determining the Anisotropic Conductivity in Electrodynamic Equations
}

\author{
Corresponding Member of the RAS V. G. Romanov ${ }^{a, b}, *$ \\ Received November 3, 2020; revised December 2, 2020; accepted December 7, 2020
}

\begin{abstract}
For a system of electrodynamic equations, the inverse problem of determining an anisotropic conductivity is considered. It is supposed that the conductivity is described by a diagonal matrix $\sigma(x)=$ $\operatorname{diag}\left(\sigma_{1}(x), \sigma_{2}(x), \sigma_{3}(x)\right)$ with $\sigma(x)=0$ outside of the domain $\Omega=\left\{x \in \mathbb{R}^{3}|| x \mid<R\right\}, R>0$, and the permittivity $\varepsilon$ and the permeability $\mu$ of the medium are positive constants everywhere in $\mathbb{R}^{3}$. Plane waves coming from infinity and impinging on an inhomogeneity localized in $\Omega$ are considered. For the determination of the unknown functions $\sigma_{1}(x), \sigma_{2}(x)$, and $\sigma_{3}(x)$, information related to the vector of electric intensity is given on the boundary $S$ of the domain $\Omega$. It is shown that this information reduces the inverse problem to three identical problems of X-ray tomography.
\end{abstract}

Keywords: Maxwell equations, anisotropy, conductivity, plane waves, inverse problem, tomography DOI: $10.1134 / \mathrm{S} 1064562421010099$

Consider the nonstationary Maxwell equations

$$
\begin{gathered}
\operatorname{curl} \mathbf{H}=\varepsilon \mathbf{E}_{t}+\sigma(x) \mathbf{E}, \\
\operatorname{curl} \mathbf{E}=-\mu \mathbf{H}_{t}, \quad \operatorname{div} \mathbf{H}=0 .
\end{gathered}
$$

Here, $\mathbf{E}=\left(E_{1}, E_{2}, E_{3}\right)$ and $\mathbf{H}=\left(H_{1}, H_{2}, H_{3}\right)$ are the electric and magnetic field strengths, $\sigma(x)=$ $\operatorname{diag}\left(\sigma_{1}(x), \sigma_{2}(x), \sigma_{3}(x)\right)$ is a positive semidefinite diagonal matrix, and $\varepsilon$ and $\mu$ are positive constants. Assume that $\sigma(x)=0$ outside the domain $\Omega=$ $\left\{x \in \mathbb{R}^{3}|| x \mid<R\right\}$, where $R>0$.

Let $c=\frac{1}{\sqrt{\varepsilon \mu}}$ denote the velocity of propagation of electromagnetic waves. Let $v=\left(v_{1}, v_{2}, v_{3}\right),|v|=1$, and $\mathbf{j}$ be the unit vector orthogonal to $v$, i.e., $\mathbf{j} \cdot v=0$.

For Maxwell's equations (1) in a homogeneous medium $(\sigma(x)=0)$, there exist solutions of the form

$$
\begin{gathered}
\mathbf{E}^{0}(x, t, v, \mathbf{j})=\mathbf{j} f\left(t+t_{0}-\frac{x \cdot v}{c}\right), \\
\mathbf{H}^{0}(x, t, v, \mathbf{j})=\frac{v \times \mathbf{j}}{\mu c} f\left(t+t_{0}-\frac{x \cdot v}{c}\right),
\end{gathered}
$$

\footnotetext{
${ }^{a}$ Sobolev Institute of Mathematics, Siberian Branch, Russian Academy of Sciences, Novosibirsk, 630090 Russia

${ }^{b}$ Mathematical Center in Akademgorodok, Novosibirsk State University, Novosibirsk, 630090 Russia

*e-mail:romanov@math.nsc.ru
}

where $t_{0}=\min _{x \in \Omega} \frac{x \cdot v}{c}=-\frac{R}{c}$ and $f(t)$ is an arbitrary generalized function. Each such solution represents a plane wave propagating in the direction of the vector $v$ and is a weak solution of Maxwell's equations for a homogeneous medium.

Consider the Cauchy problem for an anisotropic medium:

$$
\begin{gathered}
\operatorname{curl} \mathbf{H}=\varepsilon \mathbf{E}_{t}+\sigma(x) \mathbf{E}, \quad \operatorname{curl} \mathbf{E}=-\mu \mathbf{H}_{t}, \\
\left.\mathbf{E}\right|_{t<0}=\mathbf{E}^{0}(x, t, v, \mathbf{j}),\left.\quad \mathbf{H}\right|_{t<0}=\mathbf{H}^{0}(x, t, v, \mathbf{j}),
\end{gathered}
$$

where $\mathbf{E}^{0}(x, t, v, \mathbf{j})$ and $\mathbf{H}^{0}(x, t, v, \mathbf{j})$ are defined by formulas (2) and $f(t)$ is a smooth function such that $f(t) \equiv 0$ for $t \leq 0$ and $f(+0) \neq 0$. Thus, $\mathbf{E}(x, t, v, \mathbf{j})=0$ and $\mathbf{H}(x, t, v, \mathbf{j})=0$ for all $x \cdot \boldsymbol{v} \geq c t_{0}$ and $t<0$. Let $\Sigma(v)=:\left\{x \in \mathbb{R}^{3} \mid x \cdot v=c t_{0}\right\}$ be the plane corresponding to the front of a plane wave at the time $t=0$, when this front touches the domain $\Omega$.

Let $S=\left\{x \in \mathbb{R}^{3}|| x \mid=R\right\}$ be the boundary of $\Omega$ and $S^{+}(v)=\{x \in S \mid x \cdot v>0\}$ be its shadow part with respect to light propagating in the direction $v$.

Below, problem (3) is considered for three different vectors $\mathbf{j}^{k}, k=1,2,3$, and for corresponding orthogonal vectors $v^{k}$ depending on the angular parameter $\varphi$, namely, 


$$
\begin{array}{lll}
\mathbf{j}^{1}=(1,0,0), & v^{1}(\varphi)=(0, \cos \varphi, \sin \varphi), & \varphi \in[0, \pi], \\
\mathbf{j}^{2}=(0,1,0), & v^{2}(\varphi)=(\cos \varphi, 0, \sin \varphi), & \varphi \in[0, \pi],(4) \\
\mathbf{j}^{3}=(0,0,1), & v^{3}(\varphi)=(\cos \varphi, \sin \varphi, 0), & \varphi \in[0, \pi] .
\end{array}
$$

Inverse problem. Find $\sigma(x)$ from functions $E_{k}(x, t$, $\left.v^{k}(\varphi), \mathbf{j}^{k}\right), \quad k=1,2,3$, given for all $x \in S^{+}\left(v^{k}(\varphi)\right)$, $\varphi \in[0, \pi]$, and $t \in\left[0, T_{k}(x, \varphi)\right]$, where $T_{k}(x, \varphi)=$ $\frac{x \cdot v^{k}(\varphi)}{c}-t_{0}+\delta_{0}$ and $\delta_{0}>0$ is an arbitrary number (possibly small). In other words, the task is to find $\sigma(x)$ from the given functions

$$
\begin{gathered}
F_{k}(x, t, \varphi)=E_{k}\left(x, t, v^{k}(\varphi), \mathrm{j}^{k}\right), \quad k=1,2,3, \\
x \in S^{+}\left(v^{k}(\varphi)\right), \quad \varphi \in[0, \pi], \quad t \in\left[0, T_{k}(x, \varphi)\right] .
\end{gathered}
$$

For stationary electrodynamic equations, inverse problems of determining conductivity representing a one-variable function were studied by Tikhonov [1-4] and Cagniard [5]. For nonstationary equations, the theory of inverse electrodynamic problems based on the full system of Maxwell equations was developed in [6-8]. The problem of determining the permittivity of an anisotropic medium was considered in [9]. Additionally, phaseless inverse problems of determining permittivity from the magnitude of the electric or magnetic component of a stationary electromagnetic field were studied (see [10] and the review article in [11]).

The following result holds for the inverse problem stated above.

Theorem 1. Suppose that the matrix $\sigma(x)$ belongs to $C^{2}\left(\mathbb{R}^{3}\right)$ and vanishes outside $\Omega$, while the function $f(t)$ has the form $f(t)=\hat{f}(t) \theta_{0}(t)$, where $\hat{f}(t)$ is a smooth function of class $C^{2}[0, \infty), \hat{f}(0) \neq 0$, and $\theta_{0}(t)$ is the Heaviside step function, i.e., $\theta_{0}(t)=1$ for $t \geq 0$ and $\theta_{0}(t)=0$ for $t<0$. Then all elements of $\sigma(x)$ in $\Omega$ are uniquely determined by data (5).

The study of the inverse problem is based on analyzing the structure of the solution to problem (3). In this case, it is convenient to use an integro-differential equation for the vector $\mathbf{E}(x, t, v, \mathbf{j})$. To derive it, we apply the curl operator to the second equation in (3) and use the first equation to eliminate the emerging term curl $\mathbf{H}_{t}$. Then we obtain the equation

$$
(-\Delta+\nabla \operatorname{div}) \mathbf{E}=-\mu \varepsilon \mathbf{E}_{t t}-\mu \sigma(x) \mathbf{E}_{t} .
$$

Computing $\operatorname{div} \mathbf{E}$ with the help of the first equation in (3) yields

$$
\operatorname{div} \mathbf{E}(x, t, v, \mathbf{j})=-\frac{1}{\varepsilon} \operatorname{div} \int_{-\infty}^{t} \sigma(x) \mathbf{E}(x, \tau, \nu, \mathbf{j}) d \tau .
$$

It follows from (6), (7), and (3) that the function $\mathbf{E}$ is a solution of the Cauchy problem

$$
\begin{gathered}
c^{-2} \mathbf{E}_{t t}-\Delta \mathbf{E}+\mu \sigma(x) \mathbf{E}_{t} \\
-\frac{1}{\varepsilon} \nabla \operatorname{div} \int_{-\infty}^{t} \sigma(x) \mathbf{E}(x, \tau, \nu, \mathbf{j}) d \tau=0, \\
\mathbf{E}_{t<0}=\mathbf{E}^{0}(x, t, v, \mathbf{j}) .
\end{gathered}
$$

The following result holds for problem (8).

Theorem 2. Suppose that the matrix $\sigma(x)$ and the function $f(t)$ satisfy the conditions of Theorem 1 . Then the function $\mathbf{E}(x, t, v, \mathbf{j})$ for $t \geq 0$ can be represented in the form

$$
\begin{gathered}
\mathbf{E}(x, t, v, \mathbf{j})=\alpha(x, v, \mathbf{j}) \theta_{0}\left(t+t_{0}-\frac{x \cdot v}{c}\right) \\
+\hat{\mathbf{E}}(x, t, v, \mathbf{j}) \theta_{1}\left(t+t_{0}-\frac{x \cdot v}{c}\right),
\end{gathered}
$$

where $\theta_{1}(t)=t \theta_{0}(t)$, the function $\alpha(x, v, \mathbf{j})$ is a solution of the Cauchy problem

$$
\begin{gathered}
\frac{2}{c}(v \cdot \nabla) \alpha+\mu \sigma(x) \alpha-\frac{1}{\varepsilon c^{2}} v((\sigma(x) \alpha) \cdot v)=0, \\
\left.\alpha\right|_{x \cdot v=c t_{0}}=\mathbf{j} \hat{f}(0),
\end{gathered}
$$

and $\hat{\mathbf{E}}(x, t, v, \mathbf{j})$ is a bounded function of $x$ and $t$ for $t \in\left[\frac{x \cdot v}{c}-t_{0}, T\right]$ for any $T>0$.

Equation (10) is derived by substituting representation (9) into Eq. (8) and equating the coefficients of $\delta\left(t+t_{0}-\frac{x \cdot v}{c}\right)$ to zero, while the initial data for the function $\alpha(x, v, \mathbf{j})$ follow from the initial data for $\mathbf{E}(x, t, v, \mathbf{j})$. The function $\alpha(x, v, \mathbf{j})$ is the amplitude of $\mathbf{E}(x, t, v, \mathbf{j})$ at the electromagnetic wave front, i.e., at $t=\frac{x \cdot v}{c-t_{0}}$. Equation (10) is a vector ordinary differential equation along any ray $x=x^{0}+s v, s \in \mathbb{R}^{1}$, starting at the arbitrary point $x^{0} \in \mathbb{R}^{3}$. For case (4), the components of the vectors $\alpha\left(x, v^{k}(\varphi), \mathbf{j}^{k}\right)=$ $\left(\alpha_{1}\left(x, u^{k}(\varphi), \mathbf{j}^{k}\right), \alpha_{2}\left(x, v^{k}(\varphi), \mathbf{j}^{k}\right), \alpha_{3}\left(x, v^{k}(\varphi), \mathbf{j}^{k}\right)\right.$ can be computed in explicit form, namely,

$$
=\hat{f}(0) \exp \left(-\frac{\mu c}{2} \int_{0}^{\infty} \sigma_{k}\left(x-s v^{k}(\varphi)\right) d s\right), \quad k=1,2,3 .
$$

The same components can be computed using data (5) of the inverse problem:

$$
\begin{gathered}
\alpha_{k}\left(x, v^{k}(\varphi), \mathbf{j}^{k}\right)=g_{k}(x, \varphi)=: \lim _{t \rightarrow\left(x \cdot v^{k}(\varphi)\right) / c-t_{0}} F_{k}(x, t, \varphi), \\
x \in S^{+}\left(v^{k}(\varphi)\right), \quad \varphi \in[0, \pi], \quad k=1,2,3 .
\end{gathered}
$$


These formulas imply that the integrals

$\int_{0}^{\infty} \sigma_{k}\left(x-s v^{k}(\varphi)\right) d s=-\frac{2}{\mu c} \ln \frac{g_{k}(x, \varphi)}{\hat{f}(0)}, \quad k=1,2,3$,

are known for all $x \in S^{+}\left(v^{k}(\varphi)\right)$ and $\varphi \in[0, \pi]$.

Thus, for each $k=1,2,3$, the right-hand side of (11) is known along any straight line intersecting $\Omega$ in the directionv $v^{k}(\varphi)$. By varying $\varphi$, we conclude that, in each section of $\Omega$ by the plane $x_{k}=$ const, the integrals along all possible straight lines lying in this plane are known. As a result, we obtain an X-ray tomography problem for determining $\sigma_{k}(x), k=1,2,3$. It is well known that this problem is uniquely solvable (see [12-14]). This implies Theorem 1 on the uniqueness of a solution to the inverse problem and an algorithm for its solution.

\section{FUNDING}

This work was supported by the Mathematical Center in Akademgorodok at Novosibirsk State University (contract no. 075-15-2019-1613 with the Ministry of Science and Higher Education of the Russian Federation).

\section{OPEN ACCESS}

This article is distributed under the terms of the Creative Commons Attribution 4.0. International Public License (http://creativecommons.org/licenses/by/4.0/), which permits unrestricted use, distribution, and reproduction in any medium provided you give appropriate credit to the original author(s) and the source, provide a link to the Creative Commons license, and indicate if changes were made.

\section{REFERENCES}

1. A. N. Tikhonov, Izv. Akad. Nauk SSSR, Ser. Geogr. Geofiz. 10 (3), 213-231 (1946).

2. A. N. Tikhonov, Dokl. Akad. Nauk SSSR 60 (5), 797800 (1949).

3. A. N. Tikhonov, Dokl. Akad. Nauk SSSR 73 (2), 295297 (1950).

4. A. N. Tikhonov, USSR Comput. Math. Math. Phys. 5 (3), 207-211 (1965).

5. L. Cagniard, Geophysics 18 (3), 605-635 (1953).

6. V. G. Romanov and S. I. Kabanikhin, Inverse Problems in Geoelectrics (Nauka, Moscow, 1991) [in Russian].

7. A. L. Karchevsky, J. Inv. Ill-Posed Probl. 17 (4), 385402 (2009).

8. V. G. Romanov, Sib. Math. J. 52 (4), 682-695 (2011).

9. V. G. Romanov, Comput. Math. Math. Phys. 60 (6), 1037-1044 (2020).

10. A. L. Karchevsky and V. A. Dedok, J. Appl. Ind. Math. 12 (3), 470-478 (2018).

11. V. G. Romanov, Comput. Math. Math. Phys. 60 (6), 1045-1062 (2020).

12. S. Helgason, The Radon Transform (Birkhäuser, Boston, 1980).

13. F. Natterer, The Mathematics of Computerized Tomography (SIAM, Philadelphia, PA, 2001).

14. D. Finch, Inverse Probl. 2 (2), 197-203 (1986).

Translated by I. Ruzanova 